\title{
SIGN PATTERNS THAT REQUIRE OR ALLOW POWER-POSITIVITY*
}

\author{
MINERVA CATRAL ${ }^{\dagger}$, LESLIE HOGBEN H. D. D. OLESKY $^{\S}$, AND \\ P. VAN DEN DRIESSCHE
}

\begin{abstract}
A matrix $A$ is power-positive if some positive integer power of $A$ is entrywise positive. A sign pattern $\mathcal{A}$ is shown to require power-positivity if and only if either $\mathcal{A}$ or $-\mathcal{A}$ is nonnegative and has a primitive digraph, or equivalently, either $\mathcal{A}$ or $-\mathcal{A}$ requires eventual positivity. A sign pattern $\mathcal{A}$ is shown to be potentially power-positive if and only if $\mathcal{A}$ or $-\mathcal{A}$ is potentially eventually positive.
\end{abstract}

Key words. Power-positive matrix, Eventually positive matrix, Requires power-positivity, Potentially power-positive, Potentially eventually positive, Sign pattern.

AMS subject classifications. (2010) 15B48, 15B35, 05C50, 15A18.

1. Introduction. A matrix $A \in \mathbb{R}^{n \times n}$ is called power-positive $[2,10]$ if there is a positive integer $k$ such that $A^{k}$ is entrywise positive $\left(A^{k}>0\right)$. Note that if $A$ is a power-positive matrix, then $-A$ is also power-positive, because $A^{k}>0$ implies $(-A)^{2 k}>0$. If there is an odd positive integer $k$ such that $A^{k}>0$, then $A$ is called power-positive of odd exponent. Power-positive matrices have applications to the study of stability of competitive systems in economics; see, e.g., [7, 8, 9]. A real square matrix $A$ is eventually positive if there exists a positive integer $k_{0}$ such that $A^{k}>0$ for all $k \geq k_{0}$. An eventually positive matrix and its negative are both obviously power-positive.

A sign pattern matrix (or sign pattern) is a matrix having entries in $\{+,-, 0\}$. For a real matrix $A, \operatorname{sgn}(A)$ is the sign pattern having entries that are the signs of the corresponding entries in $A$. If $\mathcal{A}$ is an $n \times n$ sign pattern, the sign pattern class (or qualitative class) of $\mathcal{A}$, denoted $\mathcal{Q}(\mathcal{A})$, is the set of all $A \in \mathbb{R}^{n \times n}$ such that

* Received by the editors November 12, 2009. Accepted for publication February 18, 2010. Handling Editor: Michael J. Tsatsomeros.

$\dagger$ Department of Mathematics, Iowa State University, Ames, IA 50011, USA (mrcatral@iastate.edu).

${ }_{\ddagger}^{\ddagger}$ Department of Mathematics, Iowa State University, Ames, IA 50011, USA (lhogben@iastate.edu) \& American Institute of Mathematics, 360 Portage Ave, Palo Alto, CA 94306 (hogben@aimath.org).

$\S$ Department of Computer Science, University of Victoria, Victoria, BC V8W 3P6, Canada (dolesky@cs.uvic.ca).

"Department of Mathematics and Statistics, University of Victoria, Victoria, BC V8W 3R4, Canada (pvdd@math.uvic.ca). 
$\operatorname{sgn}(A)=\mathcal{A}$.

If $\mathcal{P}$ is a property of a real matrix, then a sign pattern $\mathcal{A}$ requires $\mathcal{P}$ if every real matrix $A \in \mathcal{Q}(\mathcal{A})$ has property $\mathcal{P}$, and $\mathcal{A}$ allows $\mathcal{P}$ or is potentially $\mathcal{P}$ if there is some $A \in \mathcal{Q}(\mathcal{A})$ that has property $\mathcal{P}$. Sign patterns that require eventual positivity have been characterized in [4], and sign patterns that allow eventual positivity have been studied in [1]. Here we characterize patterns that require power-positivity (Theorem 2.6 and Corollary 2.7) and show that a sign pattern $\mathcal{A}$ allows power-positivity if and only if $\mathcal{A}$ or $-\mathcal{A}$ allows eventual positivity (Theorem 3.1).

1.1. Definitions and notation. Let $\mathcal{A}=\left[\alpha_{i j}\right]$ and $\hat{\mathcal{A}}=\left[\hat{\alpha}_{i j}\right]$ be sign patterns. If $\alpha_{i j} \neq 0$ implies $\alpha_{i j}=\hat{\alpha}_{i j}$, then $\mathcal{A}$ is a subpattern of $\hat{\mathcal{A}}$. For a sign pattern $\mathcal{A}=\left[\alpha_{i j}\right]$, the positive part of $\mathcal{A}$ is $\mathcal{A}^{+}=\left[\alpha_{i j}^{+}\right]$where $\alpha_{i j}^{+}$is + if $\alpha_{i j}=+$ and 0 if $\alpha_{i j}=0$ or $\alpha_{i j}=$ - ; the negative part of $\mathcal{A}$ is defined analogously (see [1]). Note that $\mathcal{A}^{-}=(-\mathcal{A})^{+}$. We use $[-]$ (respectively, $[+]$ ) to denote a (rectangular) sign pattern consisting entirely of negative (respectively, positive) entries. The characteristic matrix $C_{\mathcal{A}}$ of the sign pattern $\mathcal{A}$ is the $(0,1,-1)$-matrix obtained from $\mathcal{A}$ by replacing + by 1 and - by -1 .

For an $n \times n$ sign pattern $\mathcal{A}=\left[\alpha_{i j}\right]$, the signed digraph of $\mathcal{A}$ is

$$
\Gamma(\mathcal{A})=\left(\{1, \ldots, n\},\left\{(i, j): \alpha_{i j} \neq 0\right\}\right)
$$

where an $\operatorname{arc}(i, j)$ is positive (respectively, negative) if $\alpha_{i j}=+$ (respectively, - ). Conversely, for a signed digraph $\Gamma$ on the vertices $\{1, \ldots, n\}$, the sign pattern of $\Gamma$ is $\operatorname{sgn}(\Gamma)=\left[s_{i j}\right]$ where $s_{i j}=+($ respectively, - ) if there is a positive (respectively, negative) arc from vertex $i$ to vertex $j$, and $s_{i j}=0$ otherwise. There is a one-toone correspondence between $n \times n$ sign patterns and signed digraphs on the vertices $\{1, \ldots, n\}$ and we adopt some sign pattern notation for signed digraphs. For example, $\mathcal{Q}(\Gamma)=\mathcal{Q}(\operatorname{sgn}(\Gamma))$ and $C_{\Gamma}=C_{\operatorname{sgn}(\Gamma)}$.

A signed digraph $\Gamma$ is called primitive if it is strongly connected and the greatest common divisor of the lengths of its cycles is 1 . This definition applies the standard definition of "primitive" for a digraph that is not signed to a signed digraph by ignoring the signs. Clearly for a sign pattern $\mathcal{A}, \Gamma(\mathcal{A})$ is primitive if and only if $\Gamma(-\mathcal{A})$ is primitive.

A signed subdigraph of a signed digraph is a subdigraph in which the arcs retain the signs of the original signed digraph. Let $\Gamma^{\prime}$ be a signed digraph on $n$ vertices, and let $\Gamma$ be a signed subdigraph of $\Gamma^{\prime}$ on $k$ vertices. Without loss of generality (by relabeling the vertices of $\Gamma^{\prime}$ ) assume that the vertices of $\Gamma$ are $\{1, \ldots, k\}$. For $A=\left[a_{i j}\right] \in \mathcal{Q}(\Gamma)$, define the $n \times n$ matrix $B=\left[b_{i j}\right]$ by $b_{i j}=a_{i j}$ if $(i, j) \in \Gamma^{\prime}$, and 0 otherwise. Then we call $B$ the $\Gamma^{\prime}$-embedding of $A$. Note that the sign pattern $\mathcal{B}=\operatorname{sgn}(B)$ is a subpattern of $\operatorname{sgn}\left(\Gamma^{\prime}\right)$. When a $\Gamma^{\prime}$-embedding is used in Section 
2, $\Gamma^{\prime}$ is the signed digraph $\Gamma(\mathcal{A})$ of a sign pattern $\mathcal{A}$, and we assume the necessary relabeling has been done.

1.2. Power-positive and eventually positive matrices. This subsection contains some known results about power-positive matrices and their applications. Any matrix in the sign pattern class of the sign pattern in Example 3.4 below illustrates the well known fact that there exist power-positive matrices that are not eventually positive.

An eigenvalue $\lambda_{0}$ of a matrix $A$ is strictly dominant if $\left|\lambda_{0}\right|=\rho(A)$ and for every eigenvalue $\lambda \neq \lambda_{0},|\lambda|<\left|\lambda_{0}\right|$. Every power-positive matrix $A$ has a unique real simple strictly dominant eigenvalue $\lambda_{0}$ having positive left and right eigenvectors [10]. Furthermore, if $A$ is power-positive of odd exponent, then $\lambda_{0}=\rho(A)$; otherwise, $\lambda_{0}$ may be negative. For example, any negative matrix $A$ is power-positive (with only the even powers being positive), and in this case $\lambda_{0}=-\rho(A)$. The next theorem can be deduced from [2] and the discussions on pages 43-47 in [10].

Theorem 1.1. [2, Theorem 3] If $A$ is a power-positive matrix, then either $A$ or - A has a positive simple strictly dominant eigenvalue having positive left and right eigenvectors.

Theorem 1.2. [6, p. 329] The matrix $A$ is eventually positive if and only if $A$ is power-positive of odd exponent.

Theorem 1.3. [6, Theorem 1] The matrix $A$ is eventually positive if and only if $A$ has a positive simple strictly dominant eigenvalue having positive left and right eigenvectors.

Corollary 1.4. A is a power-positive matrix if and only if either $A$ or $-A$ is eventually positive.

REMARK 1.5. Note that Corollary 1.4 is not in general true if positive is replaced by nonnegative. For example, the matrix $A=\left[\begin{array}{cc}1 & 0 \\ 0 & -1\end{array}\right]$ is a power-nonnegative matrix since $A^{2} \geq 0$, but neither $A$ nor $-A$ is eventually nonnegative.

In economics, power-positive matrices arise in the context of stability of competitive systems. Let $A=B-s I, s>0$. A system of dynamic equations [9] such as

$$
\frac{d x}{d t}=A x, x(0)=x_{0}
$$

can be interpreted as a system of price adjustment equations of competitive markets in a general equilibrium analysis. 
TheOREm 1.6. [9, Theorem 1] The competitive system (1.1) is dynamically stable if and only if $s>\rho(B)$ and $B$ satisfies one of the following conditions:

1. B is a power-positive matrix of odd exponent, or

2. $B$ is a power-positive matrix and the entries of a row or of a column of $B$ are all nonnegative.

Note that the first of the two conditions on $B$ given in Theorem 1.6 is equivalent to the eventually positivity of $B$, while the second implies that $B$ is eventually positive. Furthermore, the matrix $-A=s I-B$ in such a dynamically stable system is a pseudo- $M$-matrix as defined in [6].

2. Sign patterns that require power-positivity. In [4] it is shown that $\mathcal{A}$ requires eventual positivity if and only if $\mathcal{A}$ is nonnegative and $\Gamma(\mathcal{A})$ is primitive. In this section we use similar perturbation techniques to show that a sign pattern $\mathcal{A}$ requires power-positivity if and only either $\mathcal{A}$ or $-\mathcal{A}$ is nonnegative and $\Gamma(\mathcal{A})$ is primitive.

Observation 2.1. Let $\mathcal{A}$ be an $n \times n$ sign pattern, $\Gamma$ a signed subdigraph of $\Gamma(\mathcal{A}), A \in \mathcal{Q}(\Gamma)$ and $B$ the $\Gamma(\mathcal{A})$-embedding of $A$. Then the nonzero eigenvalues of $B$ are the nonzero eigenvalues of $A$, and the eigenvectors for the nonzero eigenvalues of $B$ are the eigenvectors of the corresponding eigenvalues of $A$, suitably embedded.

It is well known that for any matrix $A \in \mathbb{R}^{n \times n}$, the eigenvalues of $A$ are continuous functions of the entries of $A$. For a simple eigenvalue, the same is true of the eigenvector (see, for example, [5, p. 323]).

Lemma 2.2. Let $\mathcal{A}$ be an $n \times n$ sign pattern, $\Gamma$ a signed subdigraph of $\Gamma(\mathcal{A})$ and $A \in \mathcal{Q}(\Gamma)$.

1. If every nonzero eigenvalue of $A$ is simple and $A$ does not have a nonnegative eigenvector, then $\mathcal{A}$ does not require power-positivity.

2. If $A$ has a simple strictly dominant eigenvalue $\rho(A)$ that does not have a nonnegative eigenvector, then $\mathcal{A}$ does not require power-positivity.

Proof. Let $B$ be the $\Gamma(\mathcal{A})$-embedding of $A$. In either case, by Observation 2.1, the matrix $B$ retains the property of not having a nonnegative eigenvector for the relevant eigenvalue(s). Let $B(\varepsilon)=B+\varepsilon C_{\mathcal{A}}$, where $\varepsilon$ is chosen positive so that $B(\varepsilon) \in \mathcal{Q}(\mathcal{A})$, and sufficiently small so that for every simple eigenvalue of $B$, the corresponding eigenvalue and eigenvector of $B(\varepsilon)$ are small perturbations of the eigenvalue and eigenvector of $B$. In case 2, the spectral radius of $B(\varepsilon)$ is a perturbation of $\rho(A)$ because $\rho(A)$ is a strictly dominant eigenvalue. In either case, by continuity, the spectral radius of $B(\varepsilon)$ is a perturbation of one of the (nonzero) simple eigenvalues of $A$ that did not have a nonnegative eigenvector. Thus the matrix $B(\varepsilon)$ retains the 
property of not having a nonnegative eigenvector for its spectral radius, showing (by Theorem 1.1) that $B(\varepsilon)$ is not power-positive. $\square$

Lemma 2.3. Let $\mathcal{A}$ be an $n \times n$ sign pattern. If $\Gamma(\mathcal{A})$ has a signed subdigraph $\Gamma$ that is a cycle having both a positive and a negative arc, then $\mathcal{A}$ does not require power-positivity.

Proof. Suppose that the cycle $\Gamma$ is of length $k$ and has a positive $\operatorname{arc}(p, q)$ and a negative arc $(r, s)$. Note that the characteristic polynomial of $C_{\Gamma}$ is $p_{C_{\Gamma}}(x)=x^{k} \pm 1$, so the eigenvalues of $C_{\Gamma}$ are all nonzero and simple. Furthermore, no eigenvector can have a zero coordinate, so any nonnegative eigenvector must be positive. Suppose that $C_{\Gamma}$ has a positive eigenvector $x=\left[x_{i}\right]$ corresponding to an eigenvalue $\lambda$. Then the equation $C_{\Gamma} x=\lambda x$ gives

$$
x_{q}=\lambda x_{p} \text { and }-x_{s}=\lambda x_{r} .
$$

As $x_{p}, x_{q}>0$, it follows that $\lambda>0$, but on the other hand, $x_{r}, x_{s}>0$ implies that $\lambda<$ 0 , a contradiction. Thus, $C_{\Gamma}$ cannot have a nonnegative eigenvector corresponding to a nonzero eigenvalue. The result then follows from the first statement in Lemma 2.2.

Lemma 2.4. Let $\mathcal{A}$ be an $n \times n$ sign pattern. If $\Gamma(\mathcal{A})$ contains a figure-eight signed subdigraph $\Gamma(s, t)=\Gamma_{s} \cup \Gamma_{t}$ (see Figure 2.1), where $\Gamma_{s}$ is a cycle of length $s \geq 2$ with all arcs signed positively and $\Gamma_{t}$ is a cycle of length $t \geq 2$ with all arcs signed negatively, and $\Gamma_{s}$ and $\Gamma_{t}$ intersect in a single vertex, then $\mathcal{A}$ does not require power-positivity.

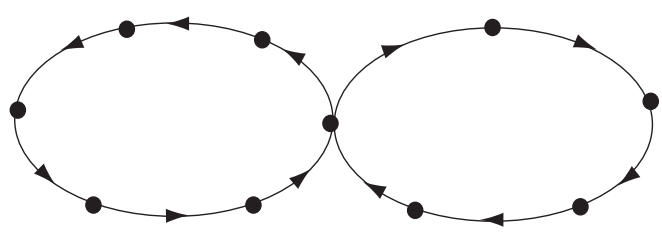

FIG. 2.1. The figure-eight $\Gamma(5,6)$

Proof. Without loss of generality, let $2 \leq s \leq t$. If $s<t$ or $s=t$ is even, the characteristic polynomial of $C_{\Gamma(s, t)}$ is

$$
p_{C_{\Gamma(s, t)}}(x)=x^{s-1} g(x), \text { where } g(x)=x^{t}-x^{t-s}+(-1)^{t+1} .
$$

Note that $g(x)$ and $g^{\prime}(x)$ have no common roots, so every nonzero eigenvalue of $C_{\Gamma(s, t)}$ is simple. Furthermore, as in the proof of Lemma 2.3, the cyclic nature of the digraph $\Gamma$ prevents any zeros in an eigenvector for a nonzero eigenvalue of $C_{\Gamma(s, t)}$, and the opposite signs prevent a positive eigenvector for a nonzero eigenvalue. The result now follows from the first statement in Lemma 2.2. 
For the case $s=t$, where $s$ is odd, let $A \in \mathcal{Q}(\Gamma(s, s))$ be obtained from $C_{\Gamma(s, s)}$ by replacing one entry equal to 1 (in the positive cycle) by 2 . Then $p_{A}(x)=x^{s-1}\left(x^{s}-1\right)$ and the result follows by the same argument as above. $\mathrm{Q}$

COROLlary 2.5. If $\mathcal{A}$ requires power-positivity, then all off-diagonal entries are nonnegative, or all off-diagonal entries are nonpositive.

Proof. If $\mathcal{A}$ requires power-positivity, then $\Gamma(\mathcal{A})$ is strongly connected and thus every arc in $\Gamma(\mathcal{A})$ lies in a cycle. Suppose that $\mathcal{A}$ has both a positive and a negative off-diagonal entry. Then $\Gamma(\mathcal{A})$ has a positive and a negative arc that lie on the same cycle, or $\Gamma(\mathcal{A})$ has two different cycles with arcs of opposite sign that intersect at a vertex. Lemma 2.3 or Lemma 2.4 implies that $\mathcal{A}$ does not require power-positivity.

THEOREM 2.6. The sign pattern $\mathcal{A}$ requires power-positivity if and only if either $\mathcal{A}$ or $-\mathcal{A}$ is nonnegative and $\Gamma(\mathcal{A})$ is primitive.

Proof. Assume that $\mathcal{A}$ requires power-positivity. Then $\Gamma(\mathcal{A})$ is strongly connected. By Corollary 2.5, the off-diagonal entries are either all nonnegative or all nonpositive. Suppose that there is a diagonal entry of opposite sign from the nonzero off-diagonal entries. Without loss of generality, suppose that the off-diagonal entries are nonpositive and that the $(1,1)$ entry of $\mathcal{A}$ is + . Let $\Gamma$ be a signed subdigraph of $\Gamma(\mathcal{A})$ consisting of a cycle of length at least two that includes vertex 1 and the loop at vertex 1. Consider $A=C_{\Gamma}+2 E_{11} \in \mathcal{Q}(\Gamma)$, where $E_{11}$ has $(1,1)$ entry equal to one and zeros elsewhere. By Gershgorin's Theorem applied to $A$, there is a unique (necessarily real) eigenvalue $\rho$ in the unit disk centered at 3 , and all other eigenvalues are in the unit disk centered at the origin, so $\rho=\rho(A)$ is simple and strictly dominant. Furthermore, no eigenvector of $A$ can have a zero coordinate. But the negative cycle entries do not allow a positive eigenvector for a positive eigenvalue. Thus by the second statement of Lemma $2.2, \mathcal{A}$ does not require power-positivity, a contradiction. Thus either $\mathcal{A}$ or $-\mathcal{A}$ is nonnegative, and so $\Gamma(\mathcal{A})$ must be primitive $[3$, Theorem 3.4.4]. The converse is clear.

COROLlaRY 2.7. The sign pattern $\mathcal{A}$ requires power-positivity if and only if either $\mathcal{A}$ or $-\mathcal{A}$ requires eventual positivity.

Proof. The necessity follows from Theorem 2.6 and [4, Theorem 2.3] and the sufficiency is clear.

3. Sign patterns that allow power-positivity. A square sign pattern $\mathcal{A}$ is called potentially power-positive (PPP) if there exists an $A \in \mathcal{Q}(\mathcal{A})$ that is powerpositive. If $A \in \mathcal{Q}(\mathcal{A})$ exists such that $A$ is eventually positive, then the sign pattern $\mathcal{A}$ is called potentially eventually positive (PEP) [1]. Note that $\mathcal{A}$ is $\mathrm{PPP}$ if and only if $-\mathcal{A}$ is PPP. The following characterization of PPP sign patterns follows from Corollary 1.4. 
TheOREM 3.1. The sign pattern $\mathcal{A}$ is potentially power-positive if and only if $\mathcal{A}$ or $-\mathcal{A}$ is potentially eventually positive.

Recall that $\mathcal{A}^{+}$is the positive part of $\mathcal{A}$. Theorem 2.1 of [1] and Theorem 3.1 above give the following result.

Theorem 3.2. If $\Gamma\left(\mathcal{A}^{+}\right)$or $\Gamma\left(\mathcal{A}^{-}\right)$is primitive, then $\mathcal{A}$ is potentially powerpositive.

We next provide examples, including a sign pattern $\mathcal{A}$ such that both $\mathcal{A}$ and $-\mathcal{A}$ are PPP, sign patterns $\mathcal{A}$ that are PPP but not PEP, and an irreducible sign pattern that is not PPP.

EXAMPLE 3.3. The sign pattern $\mathcal{A}=\left[\begin{array}{ccc}+ & + & - \\ - & 0 & + \\ + & - & -\end{array}\right]$ is $\mathrm{PPP}$, as is $-\mathcal{A}$, because both $\Gamma\left(\mathcal{A}^{+}\right)$and $\Gamma\left(\mathcal{A}^{-}\right)$are primitive.

EXAMPLE 3.4. The block sign pattern

$$
\mathcal{A}=\left[\begin{array}{ll}
{[-]} & {[-]} \\
{[-]} & {[+]}
\end{array}\right]
$$

(where the diagonal blocks are square and the diagonal [-] block is nonempty) is PPP, because $\Gamma\left(\mathcal{A}^{-}\right)$is primitive. However, $\mathcal{A}$ is clearly not PEP because the first row does not have a $+[6$, p. 327].

EXAMPLE 3.5. A square sign pattern $\mathcal{A}=\left[\alpha_{i j}\right]$ is a $Z$ sign pattern if $\alpha_{i j} \neq+$ for all $i \neq j$. An $n \times n Z$ sign pattern $\mathcal{A}$ with $n \geq 2$ cannot be PEP [1, Theorem 5.1], but if $\Gamma\left(\mathcal{A}^{-}\right)$is primitive, then by Corollary 3.2, $\mathcal{A}$ is PPP. For $n \geq 3$, if $\mathcal{A}$ is an $n \times n$ $Z$ sign pattern having every off-diagonal entry nonzero, then $\Gamma\left(\mathcal{A}^{-}\right)$is primitive and thus $\mathcal{A}$ is PPP.

Note that when $n=2, \mathcal{A}=\left[\begin{array}{ll}+ & - \\ - & +\end{array}\right]$ is not PPP, as in the next example, where Theorem 3.1 is used to show that a generalization of this sign pattern is not PPP.

ExAmple 3.6. Let

$$
\mathcal{A}=\left[\begin{array}{cccc}
{[+]} & {[-]} & {[+]} & \ldots \\
{[-]} & {[+]} & {[-]} & \ldots \\
{[+]} & {[-]} & {[+]} & \ldots \\
\vdots & \vdots & \vdots & \ddots
\end{array}\right]
$$

where the diagonal blocks are square and there are at least 2 diagonal blocks. Then no subpattern of $\mathcal{A}$ is PPP because by [1, Theorems 5.3 and 3.1], no subpattern of $\mathcal{A}$ or $-\mathcal{A}$ is PEP. Thus by Theorem 3.1, no subpattern of $\mathcal{A}$ is PPP. 
Acknowledgements This research is a sequel to research done at the December 2008 American Institute of Mathematics workshop, "Nonnegative Matrix Theory: Generalizations and Applications," and the authors thank AIM and NSF for their support of that workshop. The research of D.D. Olesky and P. van den Driessche was supported in part by NSERC Discovery grants.

\section{REFERENCES}

[1] A. Berman, M. Catral, L.M. DeAlba, A. Elhashash, F.J. Hall, L. Hogben, I.-J. Kim, D.D. Olesky, P. Tarazaga, M.J. Tsatsomeros, and P. van den Driessche. Sign patterns that allow eventual positivity. Electronic Journal of Linear Algebra, 19:108-120, 2010.

[2] A. Brauer. On the characteristic roots of power-positive matrices, Duke Math. J., 28:439-445, 1961.

[3] R.A. Brualdi and H.J. Ryser. Combinatorial Matrix Theory, Cambridge University Press, Cambridge, 1991.

[4] E. M. Ellison, L. Hogben, and M. J. Tsatsomeros. Sign patterns that require eventual positivity or require eventual nonnegativity. Electronic Journal of Linear Algebra, 19:98-107, 2010.

[5] G. H. Golub and C. F. Van Loan. Matrix Computations (third edition). Johns Hopkins University Press, Baltimore, 1996.

[6] C. R. Johnson and P. Tarazaga. On matrices with Perron-Frobenius properties and some negative entries. Positivity, 8:327-338, 2004.

[7] K. Okuguchi. Power-positive matrices and global stability of competitive equilibrium. Keio Economic Studies, 12:37-40, 1975.

[8] R. Sato. The stability of the competitive system which contains gross complementary goods. Review of Economic Studies, 39:495-499, 1972.

[9] R. Sato. On the stability of properties of dynamic economic systems. International Economic Review, 14:753-764, 1973.

[10] E. Seneta. Non-negative Matrices, An Introduction to Theory and Applications. George Allen and Unwin Ltd, London, 1973. 